J. Clin. Chem. Clin. Biochem.

Vol. 23, 1985, pp. 57-62

\title{
Quantitative Proteinbestimmung im Harn mit einer empfindlichen Streulichtmethode
}

\author{
Von $H$. Kirchherr und H.-W. Schiwara \\ Aus dem Labor Dr. Schiwara, Dr. von Winterfeld, Dr. Pfanzelt, Bremen
}

(Eingegangen am 26. Juli/12. Oktober 1984)

Zusammenfassung: Die von Reiber ((1980) J. Clin. Chem. Clin. Biochem. 18, 123-127) beschriebene Streulichtmethode für die Proteinbestimmung im Liquor eignet sich auch zur Messung des Gesamteiweißes im Harn. Die Meßbereiche sind 50-500 mg/l (Methode A) und 5-70 mg/l (Methode B). Konzentrationen > $150 \mathrm{mg} / \mathrm{l}$ korrelieren gut mit der Biuret- und der Coomassie-Methode $(r=0,9804$ bzw. 0,9925). Proteinkonzentrationen $<150 \mathrm{mg} / \mathrm{l}$ weisen nur mit dem empfindlichen Albumin-Ėnzymimmunoassay eine gute Korrelation auf $(r=0,9342)$. Medikamenteneinflüsse konnten für Penicillin, Gentamycinsulfat, Dihydralazinsulfat, Amoxicillin und Furosemid nicht nachgewiesen werden. Bei 52 offensichtlich gesunden Personen wurde eine Proteinausscheidung von $7-56 \mathrm{mg} / 24 \mathrm{~h}(90 \%$-Vertrauensbereich) gefunden.

\section{Quantitation of urinary protein by a sensitive nephelometric method}

Summary: The nephelometric method of Reiber ((1980) J. Clin. Chem. Clin. Biochem. 18, 123-127) for quantitation of proteins in cerebrospinal fluid was modified for urine. Protein concentrations between 50 and $500 \mathrm{mg} / \mathrm{l}(\operatorname{method} \mathrm{A})$ or between 5 and $70 \mathrm{mg} / \mathrm{l}(\mathrm{method} B)$ can be measured. Values $>150 \mathrm{mg} / \mathrm{l}$ correlate well with the Biuret- and the Coomassie method $(r=0.9804$ and $r=0.9925$, respectively). For lower concentrations, only albumin, determined by an enzyme-immuno-assay, gave a good correlation $(r=0.9342)$. The following pharmaceuticals did not influence the results: penicillin, gentamycin sulphate, dihydralazine sulphate, amoxicillin and furosemide. A reference range of $7-56 \mathrm{mg} / 24 \mathrm{~h}$ was established $(\mathrm{n}=52,90 \%$ confidence interval).

\section{Einführung}

Die Gesamtproteinbestimmung im Harn wird hauptsächlich mit der Biuret- (1) oder der CoomassieReaktion (2), seltener mit turbidimetrischen Methoden (3) durchgeführt. Keines dieser Verfahren ist ausreichend empfindlich für die zuverlässige Messung der Harnproteine im Normalbereich. Dieses ist jedoch eine wichtige Voraussetzung, um bei der diskelektrophoretischen Differenzierung von Proteinurien sehr unterschiedlicher Konzentration (Gesamteiwei $B$ im Harn 10 bis $>10.000 \mathrm{mg} / \mathrm{l}$ ) die Gele gleichmäßig beladen und vergleichbare Ergebnisse erzielen zu können. Wir haben daher die von Reiber (4) für die Liquorproteinbestimmung beschriebene Streulichtmethode auf ihre Eignung für unsere Problemstellung untersucht. Die bekannten turbidimetrischen
Methoden sind relativ ungenaue Meßverfahren ohne stabilen Streulichtendwert. Bei der Methode von Reiber dagegen durchläuft die Streulichtintensität je nach Proteinkonzentration nach wenigen Sekunden bis Minuten ein gut definierbares Maximum. Dieses maximale Streulichtsignal ist linear proportional zur Proteinkonzentration.

\section{Material und Methode \\ Reagenzien}

Trichloressigsäure p. A. (Merck, Darmstadt), Humanserumalbumin (Behringwerke, Marburg), Saures $\alpha_{1}$-Glykoprotein (Sigma, St. Louis), Myoglobin (Serva, Heidelberg), IgG (Cutter, Berkeley), Kontrollserum Precinorm U (Bochringer, Mannheim), Albustix (Ames, Paris). 
Folgende Medikamente wurden uns freundlicherweise kostenlos zur Verfügung gestellt:

Penicillin (Heyl, Berlin) Gentamycinsulfat (Merck, Darmstadt), Dihydralazinsulfat (Henning, Berlin), Amoxicillin und Furosemid (Ratiopharm, Blaubeuren).

\section{Streulichtmethode}

Etwa $1 \mathrm{ml}$ Urin wird zentrifugiert ( 2 min bei $9600 \mathrm{~g}$, EppendorfZentrifuge). Die Proteinkonzentration wird durch ScreeningTest (Albustix ${ }^{\circledR}$ ) geschätzt und der Urin gegebenenfalls entsprechend verdünnt. Die Trichloressigsäurelösung und das verwendete dest. Wasser werden durch 0,45 $\mu \mathrm{m}$ Filter (Millipore, Bedford) filtriert. Die Messung erfolgt am Beckman Immunochemistry Analyzer ( $70^{\circ}$ Vorwärtsstreuung, 400-550 nm) im Scatter Mode mit angeschlossenem Schreiber.

\section{A) Meßbereich $50-500 \mathrm{mg} / \mathrm{l}$}

In einer Rundküvette mit Rührer werden bei laufender Registrierung und eingeschaltetem Rührwerk $50 \mu \mathrm{J}$ Urin zu 550 l $2,4 \mathrm{~mol} / 1$ Trichloressigsäure pipettiert. Das maximale Streulichtsignal wird abgelesen. Der Leerwert für die Trichloressigsäure wird subtrahiert. Die Proteinkonzentration wird mit Hilfe einer Humanserumalbuminlösung (200 mg/l) als Standard ermittelt.

\section{B) Meßbereich 5-70 mg/l}

$350 \mu \mathrm{l}$ Urin werden in einer Rundküvette mit Rührer vorgelegt. Man pipettiert bei laufender Registrierung und eingeschaltetem Rührwerk $250 \mu \mathrm{l} 5,28 \mathrm{~mol} / \mathrm{l}$ Trichloressigsäure hinzu und be- stimmt das maximale Streulichtsignal. Der Leerwert wird gesondert ermittelt durch Zugabe von $250 \mu$ l dest. Wasser zu $350 \mu l$ Urin. Als Standard dient eine Humanserumalbuminlösung $(25 \mathrm{mg} / \mathrm{l})$. Falls sich Gasblasen bilden (erkennbar an stark schwankenden Scatterwerten), nimmt man die Küvette kurz aus der Halterung und entfernt die Blasen durch leichtes Klopfen gegen die Küvettenwand. Die Bildung von Gasblasen läßt sich vermeiden, wenn alkalische Ựipe zuvor neutralisiert werden.

\section{Vergleichsmethoden}

Proteinbestimmungen im Harn wurden außerdem mit der Biuretmethode (Testkombination Boehringer Mannheim) und mit Coomassie Brilliant Blue G250 (5) durchgeführt. Als Standards dienten bei der Coomassie-Methode Humanserumalbuminlösungen der Konzentrationen $10-800 \mathrm{mg} / \mathrm{l}$.

Die quantitative Bestimmung von Albumin im Harn erfolgte mit einem Doppel-Antikörper-Sandwich ELISA (6).

\section{Ergebnisse}

\section{Linearität}

Im gesamten Meßbereich besteht lineare Abhängigkeit zwischen maximaler Streulichtintensität und Proteinkonzentration (Abb. 1).
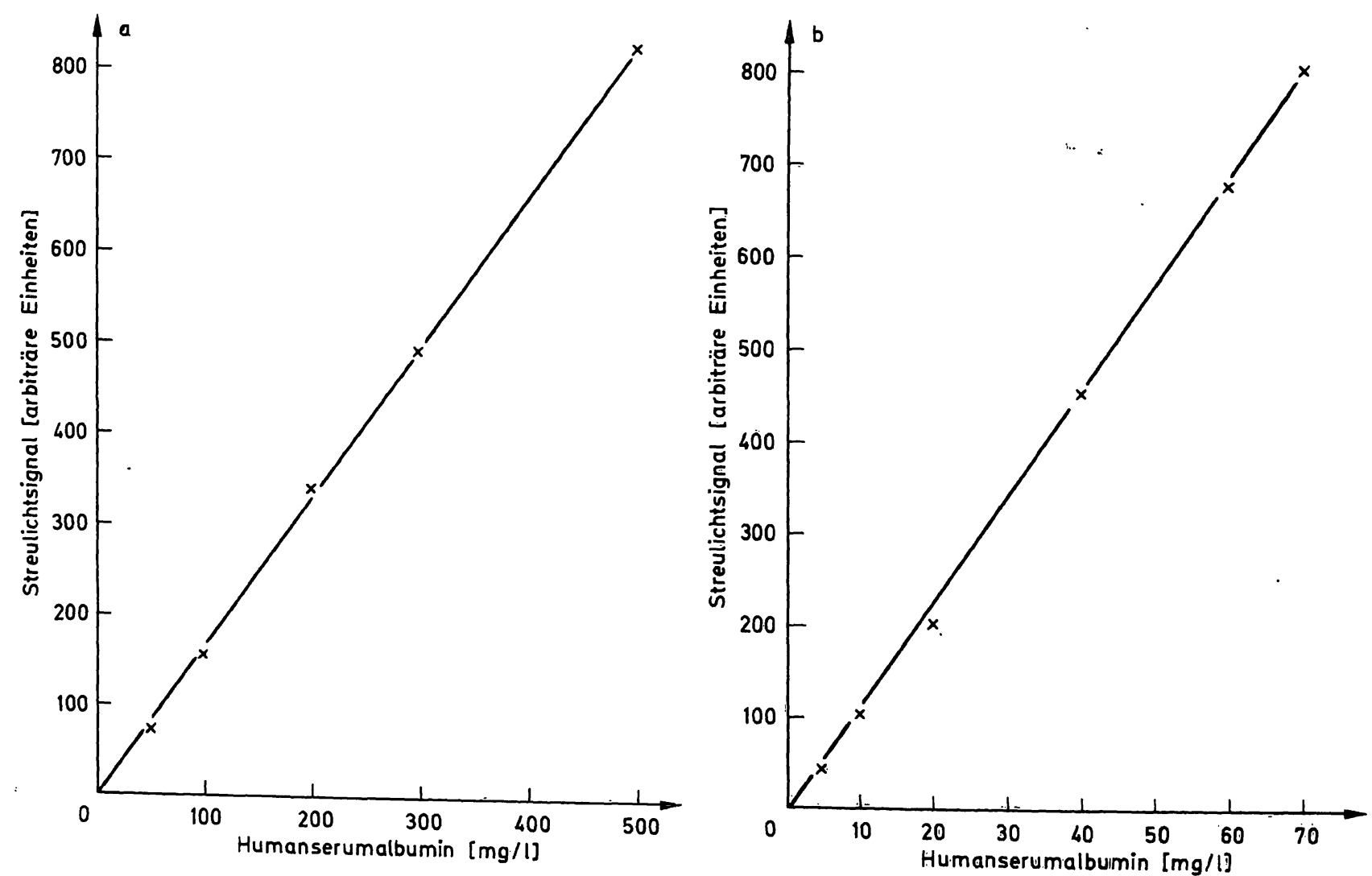

Abb.1. Standardreihe Humanserumalbumin.

a) Methode A (Meßbereich $50-500 \mathrm{mg} / \mathrm{l}), \mathrm{y}=1,66 \mathrm{x}-4,76, \mathrm{r}=0,9997$,

b) Methode B (Meßbereich $5-70 \mathrm{mg} / \mathrm{l}), \mathrm{y}=11,66 x-19,2, \mathrm{r}=0,9999$. 


\section{Empfindlichkeit}

Die Empfindlichkeit der Methode läßt sich durch Ermittlung des Diskriminierungsvermögens (7) beurteilen. Es wurden 4 Urine mit einer Proteinkonzentration von $10-15 \mathrm{mg} / \mathrm{l}$ je $11 \mathrm{mal}$ vermessen und der Mittelwert berechnet. Ein Unterschied von $1 \mathrm{mg} / \mathrm{l}$ konnte noch eindeutig diskriminiert werden. Die sich daraus ergebende Nachweisgrenze von $1 \mathrm{mg} / \mathrm{l}$ erscheint jedoch wegen der langen Meßzeiten unrealistisch. Dagegen ist eine Nachweisgrenze von $5 \mathrm{mg} / \mathrm{l}$ praktikabel (Abb. 6).

\section{Spezifität}

Verschiedene Reinproteine wurden mit der Methode untersucht. Bezogen auf Humanalbumin betrugen die maximalen Streulichtintensitäten bei einer Konzentration von $400 \mathrm{mg} / \mathrm{l}$ für IgG $100 \%$, Transferrin $81 \%$ und Myoglobin 92\% (Abb. 2). Der Korrelationskoeffizient der Standardgeraden war in allen Fällen gröBer als 0,996. Saures $\alpha_{1}$-Glykoprotein wird wie bei der Biuret-Methode nicht erfaßt. Der Einfluß von 5 gängigen Medikamenten wurde untersucht (8). Penicillin (15 Mio. i.E./1), Amoxicillin (3 g/l), Gentamycinsulfat $(0,3 \mathrm{~g} / \mathrm{l})$, Furosemid $(0,25 \mathrm{~g} / \mathrm{l})$ und Dihydralazinsulfat $(0,1 \mathrm{~g} / \mathrm{l})$ wurden einer Humanserumalbuminlösung $(200 \mathrm{mg} / \mathrm{l})$ und je 5 Patientenurinen (Konzentrationsbereich $25-6000 \mathrm{mg} / \mathrm{l}$ ) zugemischt. Ein Einfluß konnte nicht nachgewiesen werden. Die gefundene mittlere Abweichung von 2-3\% kann als statistische Streuung gewertet werden.

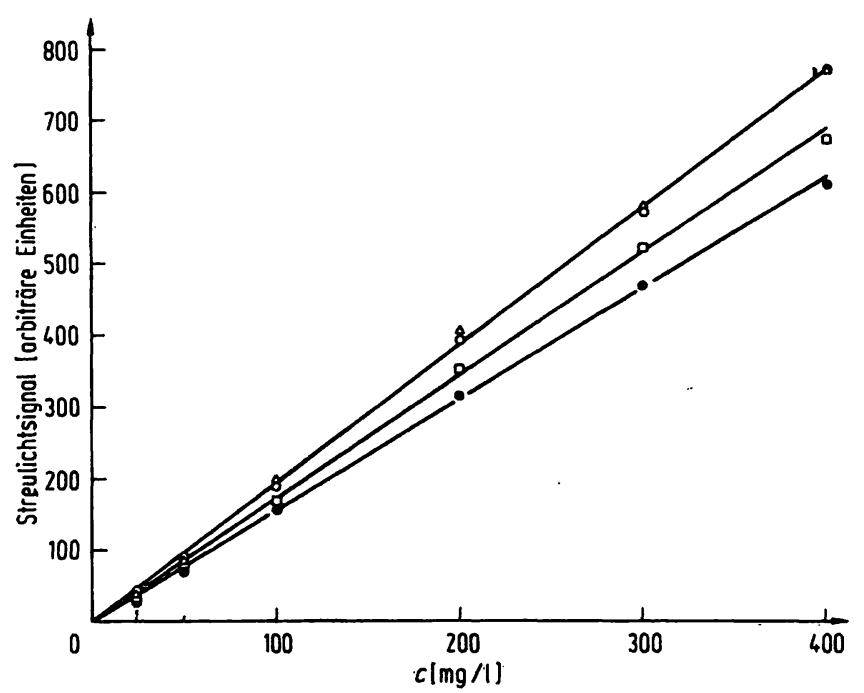

Abb. 2. Vergleich verschiedener Reinproteine im Konzentrationsbereich $25-400 \mathrm{mg} / \mathrm{l}$.

$\mathrm{O}-\mathrm{O}$ Humanserumalbumin,

$\triangle-\triangle$ IgG,

$\triangle-\square$ Myoglobin,

-0 Transferrin.
Vergleich mit anderen Methoden

116 bzw. 105 Patientenurine wurden mit der Streulicht-, der Biuret- und Coomassie-Methode untersucht (Abb. 3 und 4). Außerdem wurden in 52 Normalurinen das Gesamteiweiß mit der Streulicht- und

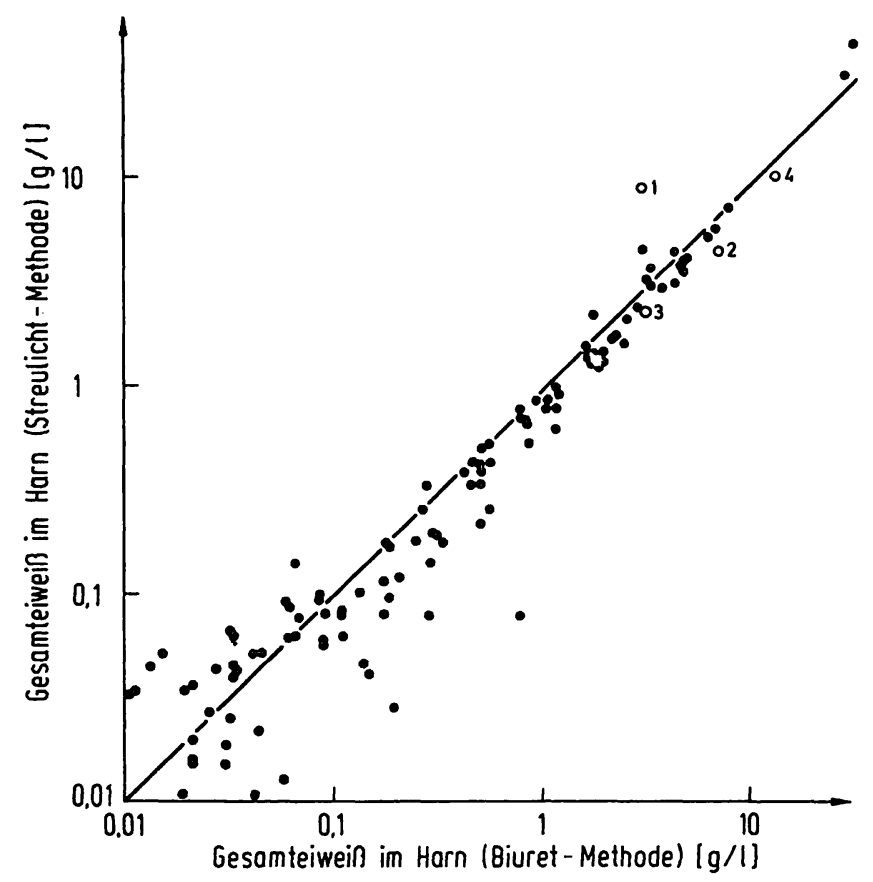

Abb. 3. Gesamteiweiß im Harn. Methodenvergleich: Streulichtmethode/Biuret.

116 Patientenurine. Die Urine mit Bence-Jones-Proteinen sind besonders gekennzeichnet $\left({ }^{\circ}\right)$.

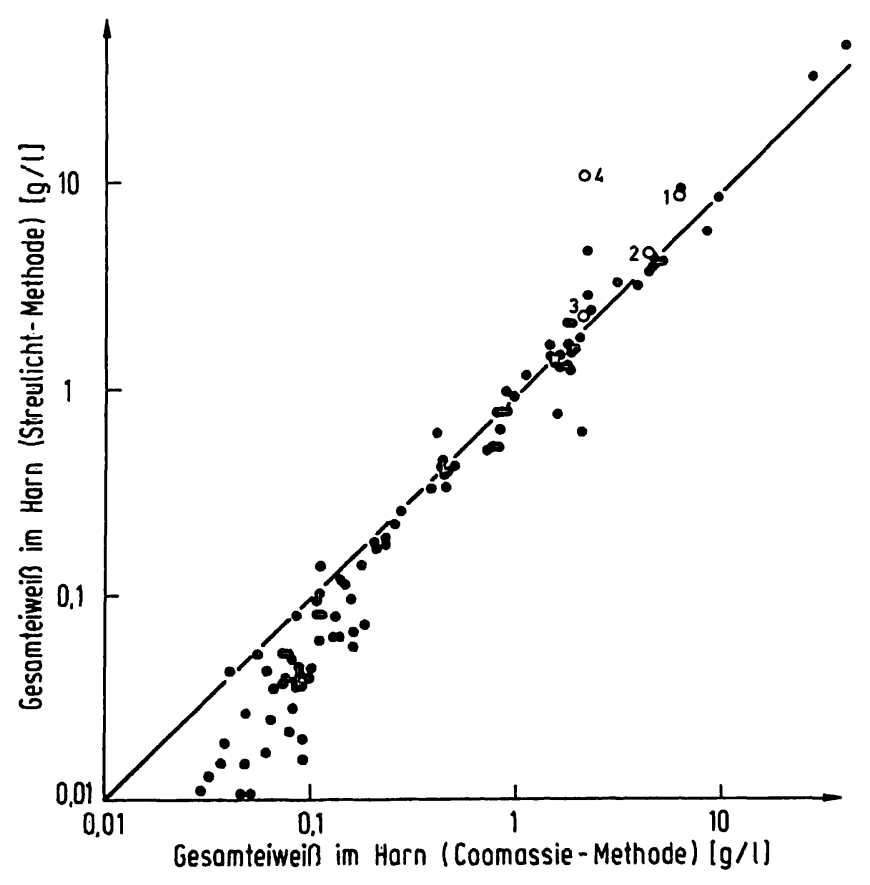

Abb. 4. Gesamteiweiß im Harn. Methodenvergleich: Streulichtmethode/Coomassie.

105 Patientenurine. Die Urine mit Bence-Jones-Proteinen sind besonders gekennzeichnet $\left({ }^{\circ}\right)$. 
der Coomassie-Methode sowie das Albumin enzymimmunologisch gemessen. Die gefundenen Werte wurden korreliert (Tab. 1). Eine gute Korrelation fand sich zwischen Streulicht- und Coomassie- sowie Streulicht- und Biuretmethode bei Proteinkonzentrationen $>150 \mathrm{mg} / \mathrm{l}$. Bei Proteinkonzentrationen $<150$ $\mathrm{mg} / \mathrm{l}$ war die Korrelation deutlich schlechter. Besser dagegen korrelierten Streulichtmethode und Albumin in diesem Konzentrationsbereich.

\section{Richtigkeit}

Die Richtigkeit wurde mit 1:200 verdünntem Precinorm U (Sollwert $51 \mathrm{~g} / \mathrm{l}$ ) ermittelt. Die mittlere Abweichung vom Sollwert an 3 Tagen $(\mathrm{n}=3)$ betrug $2,0 \%$. Mit 3 Urinen $(2,9$ und $94 \mathrm{mg} / \mathrm{l})$ wurde eine Aufstockung von Humanserumalbumin $(25,50,100$, 200 und $400 \mathrm{mg} / \mathrm{l}$ ) durchgeführt. Die Wiederfindung betrug $91-105 \%$. Der Korrelationskoeffizient der ermittelten Geraden lag über 0,999.

\section{Referenzbereich}

Bei 52 klinisch offensichtlich gesunden Personen wurden im $24 \mathrm{~h}$ Sammelurin die in Tabelle 2 dargestellten Protein- bzw. Albuminausscheidungen gefunden. Die Histogramme der Referenzwertverteilungen sind in Abbildung 5 dargestellt.

\section{Präzision}

Die Präzision in Serie $(n=21)$ wurde an jeweils drei Patientenurinen mit Methode A und B ermittelt und mit der Coomassie- und Biuretmethode verglichen (Tab. 3). Die Urine 5 und 6 wurden nicht mit der Coomassie- und Biuretmethode untersucht, da die Proteinkonzentrationen nahe oder unterhalb der in der Literatur angegebenen Nachweisgrenzen von 30 bzw. $90 \mathrm{mg} / \mathrm{l}$ liegen (5). Für die Präzision von Tag zu Tag betrug bei Verwendung von 1:200 verdünntem Precinorm U der VK $=6,5 \%(n=20, \bar{x}=277$ $\mathrm{mg} / \mathrm{l})$.

\section{Zeitbedarf}

Der Zeitaufwand für die einzelne Messung ist abhängig von der Proteinkonzentration in der Probe. In Abbildung 6 sind die Meßzeiten für Humanserumalbuminlösungen dargestellt. Bei einer Konzentration von $400 \mathrm{mg} / \mathrm{l}$ beträgt die Meßzeit etwa $30 \mathrm{~s}$, bei 20 $\mathrm{mg} / \mathrm{l}$ dagegen schon $600 \mathrm{~s}$ (Methode A). Wird die Albuminlösung mit der Konzentration $20 \mathrm{mg} / \mathrm{l} \mathrm{mit}$ der Methode B gemessen, erreicht die Streulichtintensität schon nach etwa $60 \mathrm{~s}$ ihr Maximum.
Tab.1. Lineare Regression und Korrelationskoeffizienten für Gesamtprotein (Streulicht-, Coomassie- und Biuretmethode) sowie Albumin (Enzymimmunoassay) im Harn.

\begin{tabular}{|c|c|c|c|}
\hline Methodenvergleich & Regressionsgerade & $\begin{array}{l}\text { Korr.- } \\
\text { koeff. }\end{array}$ & n \\
\hline \multicolumn{4}{|l|}{ Patientenurine } \\
\hline $\begin{array}{l}\text { Streulicht }(y), \\
\text { Coomassie }(x) \\
\text { alle Werte } \\
>150 \mathrm{mg} / 1 \\
<150 \mathrm{mg} / 1\end{array}$ & $\begin{array}{l}y=1,31 x-288,4 \\
y=1,32 x-512,3 \\
y=0,68 x-9,05\end{array}$ & $\begin{array}{l}0,9901 \\
0,9925 \\
0,7503\end{array}$ & $\begin{array}{r}105 \\
56 \\
49\end{array}$ \\
\hline $\begin{array}{l}\text { Streulicht }(y), \\
\text { Biuret }(x) \\
\text { alle Werte } \\
>150 \mathrm{mg} / 1 \\
<150 \mathrm{mg} / 1\end{array}$ & $\begin{array}{l}y=1,25 x-353,0 \\
y=1,29 x-417,4 \\
y=0,58 x+18,9\end{array}$ & $\begin{array}{l}0,9758 \\
0,9804 \\
0,7792\end{array}$ & $\begin{array}{r}116 \\
67 \\
49\end{array}$ \\
\hline \multicolumn{4}{|l|}{ Normalurine } \\
\hline $\begin{array}{l}\text { Streulicht (x), } \\
\text { Albumin (y) }\end{array}$ & $y=0,349 x-1,95$ & 0,9342 & 50 \\
\hline $\begin{array}{l}\text { Streulicht (y), } \\
\text { Coomassie (x) }\end{array}$ & $y=0,388 x+3,01$ & 0,6368 & 52 \\
\hline $\begin{array}{l}\text { Coomassie (x), } \\
\text { Albumin (y) }\end{array}$ & $y=0,1887 x-3,06$ & 0,5752 & 50 \\
\hline
\end{tabular}

Tab. 2. Referenzbereich und Mittelwerte von 52 offensichtlich gesunden Personen für Gesamtprotein (Streulicht- und Coomassiemethode) und Albumin (Enzymimmunoassay) (Ausscheidung in $\mathrm{mg} / 24 \mathrm{~h}$ ).

\begin{tabular}{|c|c|c|c|c|}
\hline Methode & Bereich & $\overline{\mathbf{x}}$ & Median & $\begin{array}{l}90 \% \text { Ver- } \\
\text { trauens= } \\
\text { bereich }\end{array}$ \\
\hline $\begin{array}{l}\text { Streulicht } \\
\text { Coomassie } \\
\text { Albumin }\end{array}$ & $\begin{array}{c}5-70 \\
14-101 \\
1,9-20,5\end{array}$ & $\begin{array}{c}23,2 \\
53,8 \\
7,61\end{array}$ & $\begin{array}{r}20,5 \\
49,5 \\
5,4\end{array}$ & $\begin{array}{c}7-56 \\
23-97 \\
2,1-16,0\end{array}$ \\
\hline
\end{tabular}

\section{Diskussion}

Die Streulichtmethode von Reiber (4) ist ein einfaches Verfahren zur quantitativen Bestimmung des Gesamteiweißes im Harn. Im Gegensatz zu der Biuret- und der Coomassie-Methode sind auch im Normalbereich zuverlässige Messungen möglich, eine wichtige Voraussetzung für die diskelektrophoretische Differenzierung von Harnproteinen. Bei Proteinurien von unter $150 \mathrm{mg} / \mathrm{l}$ findet sich eine gute Korrelation zwischen den mit der Streulichtmethode gemessenen Protein- und den enzymimmunologisch bestimmten Albuminkonzentrationen. Die Mittelwerte verhalten sich wie 3:1 (Tab. 2). Dieses Verhältnis stimmt gut mit den Angaben aus der Literatur überein, wonach der Anteil von Albumin am Gesamteiweîß in Normalurinen etwa $30 \%$ betragen soll (9). Im Konzentrationsbereich über $150 \mathrm{mg} / \mathrm{l}$ ist die Übereinstimmung mit der Biuret- und der Coomassiemethode gut. Mit unterschiedlichen Proteinen (Albựmin, Immunglobu- 


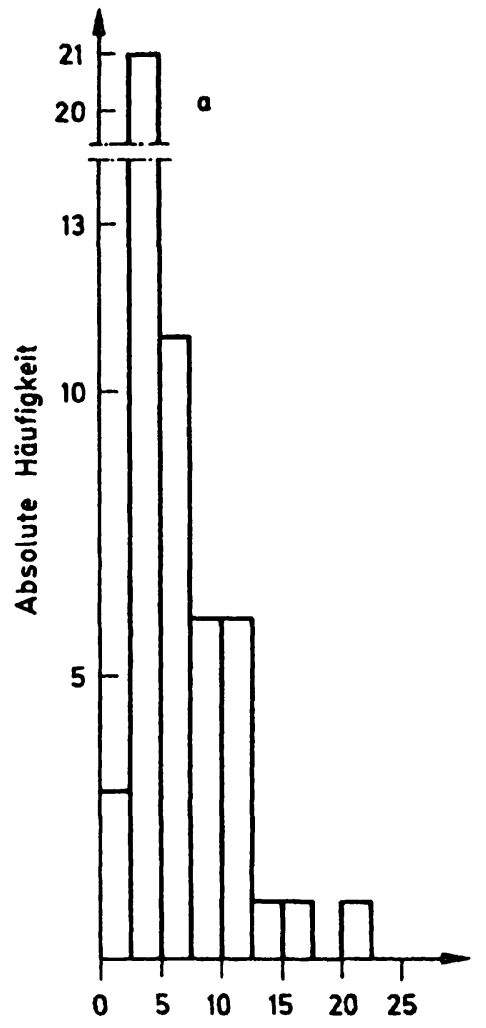

Albumin (Enzymimmunoassay) [mg/l]
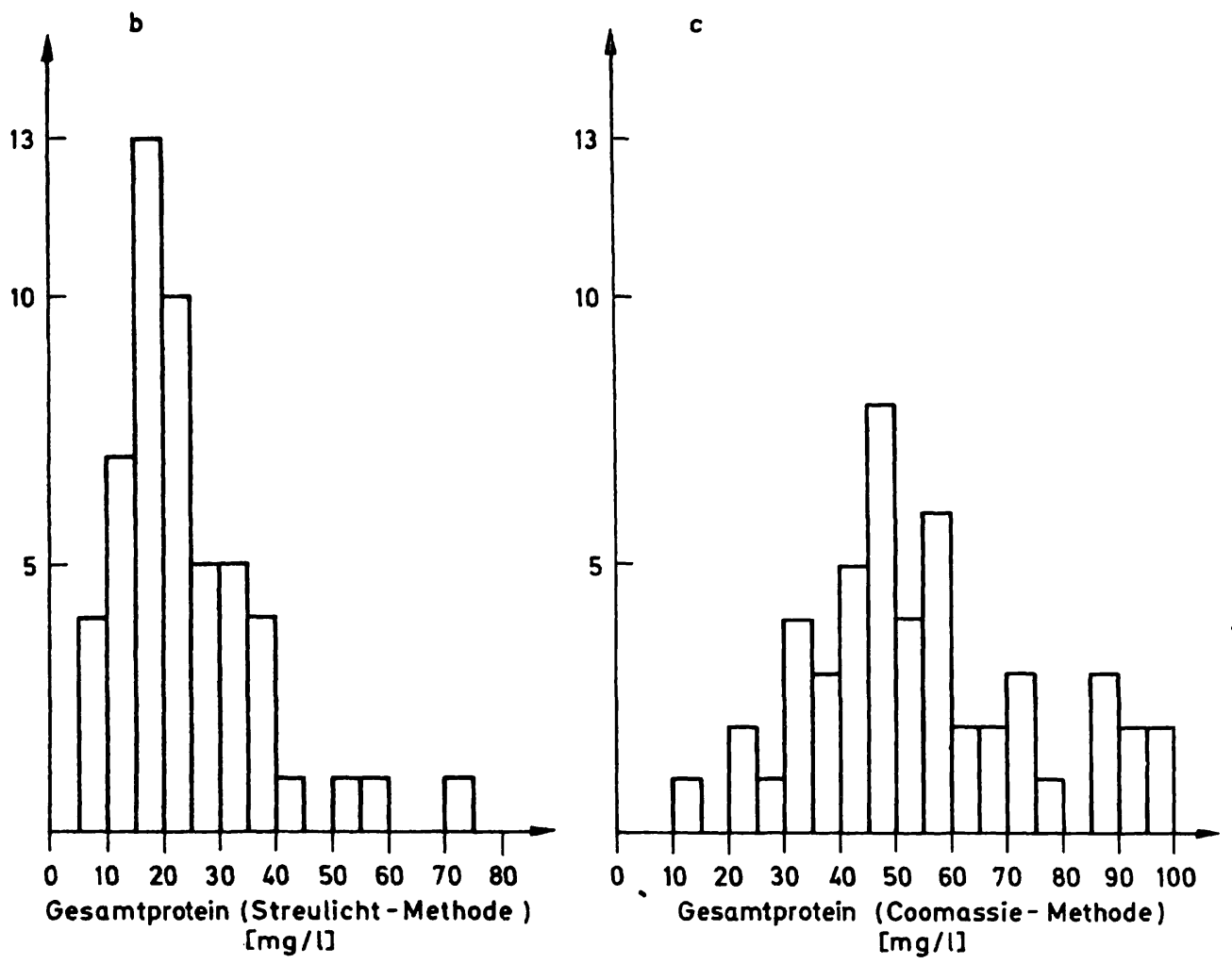

Abb. 5. Histogramm der Tabelle 2 zugrundeliegenden Einzelwerte.

a) Albumin $(n=50)$, b) Gesamtprotein, Streulichtmethode $(n=52)$, c) Gesamtprotein, Coomassie-Methode $(n=52)$.

Tab. 3. Präzision in Serie. Vergleich von Streulicht-, Coomassie- und Biuretmethode.

$$
\begin{aligned}
\overline{\mathbf{x}} & =\text { Mittelwert } \\
\mathbf{S} & =\text { Standardabweichung } \\
\text { VK } & =\text { Variationskoeffizient }
\end{aligned}
$$

\begin{tabular}{|c|c|c|c|c|c|c|}
\hline Methode & $\begin{array}{l}\text { Probe } \\
\text { Nr. }\end{array}$ & $\begin{array}{l}\overline{\mathrm{x}} \\
(\mathrm{mg} / \mathrm{l})\end{array}$ & $\begin{array}{l}x_{\min } \\
(\mathrm{mg} / \mathrm{l})\end{array}$ & $\begin{array}{l}\mathrm{x}_{\max } \\
(\mathrm{mg} / \mathrm{l})\end{array}$ & $\begin{array}{l}\mathrm{s} \\
(\mathrm{mg} / \mathrm{l})\end{array}$ & $\begin{array}{l}\text { VK } \\
(\%)\end{array}$ \\
\hline Streulicht A & $\begin{array}{l}1 \\
2 \\
3\end{array}$ & $\begin{array}{l}447 \\
187 \\
124\end{array}$ & $\begin{array}{l}438 \\
182 \\
120\end{array}$ & $\begin{array}{l}458 \\
194 \\
128\end{array}$ & $\begin{array}{l}6,42 \\
3,59 \\
2,86\end{array}$ & $\begin{array}{l}1,4 \\
1,9 \\
2,3\end{array}$ \\
\hline Streulicht B & $\begin{array}{l}4 \\
5 \\
6\end{array}$ & $\begin{array}{l}59 \\
36 \\
11\end{array}$ & $\begin{array}{l}58 \\
35 \\
10\end{array}$ & $\begin{array}{l}61 \\
37 \\
11\end{array}$ & $\begin{array}{l}0,665 \\
0,914 \\
0,291\end{array}$ & $\begin{array}{l}1,1 \\
2,5 \\
2,8\end{array}$ \\
\hline Coomassie & $\begin{array}{l}1 \\
2 \\
3 \\
4\end{array}$ & $\begin{array}{r}413 \\
200 \\
136 \\
68\end{array}$ & $\begin{array}{r}404 \\
195 \\
130 \\
64\end{array}$ & $\begin{array}{r}421 \\
205 \\
140 \\
70\end{array}$ & $\begin{array}{l}4,64 \\
3,04 \\
2,46 \\
1,72\end{array}$ & $\begin{array}{l}1,1 \\
1,5 \\
1,8 \\
2,5\end{array}$ \\
\hline Biuret & $\begin{array}{l}1 \\
2 \\
3 \\
4\end{array}$ & $\begin{array}{r}645 \\
242 \\
116 \\
73\end{array}$ & $\begin{array}{r}597 \\
209 \\
92 \\
51\end{array}$ & $\begin{array}{r}676 \\
294 \\
150 \\
90\end{array}$ & $\begin{array}{r}19,40 \\
22,64 \\
16,06 \\
9,37\end{array}$ & $\begin{array}{r}3,0 \\
9,3 \\
13,9 \\
12,8\end{array}$ \\
\hline
\end{tabular}

lin G, Transferrin und Myoglobin) werden vergleichbare Streulichtintensitätten gemessen. Bei Bence-Jones-Proteinurien sind die mit der Streulicht- und der Biuretmethode erhaltenen Werte gut vergleichbar. Mit der Coomassie-Methode lagen die Konzentrationen z. T. viel niedriger. Medikamenteneinflüsse konnten nicht nachgewiesen werden.
Die Methode hat sich in der Routinediagnostik bewährt. Nachteilig ist der relativ kleine Meßbereich, der bei den sehr unterschiedlich hohen Proteinurien $(10->10000 \mathrm{mg} / \mathrm{l})$ Wiederholungen der Messung nach Methode B oder nach entsprechender Probenverdünnung erforderlich machen kann. Das Verfahren kann zur Zeit nicht mechanisiert werden. 

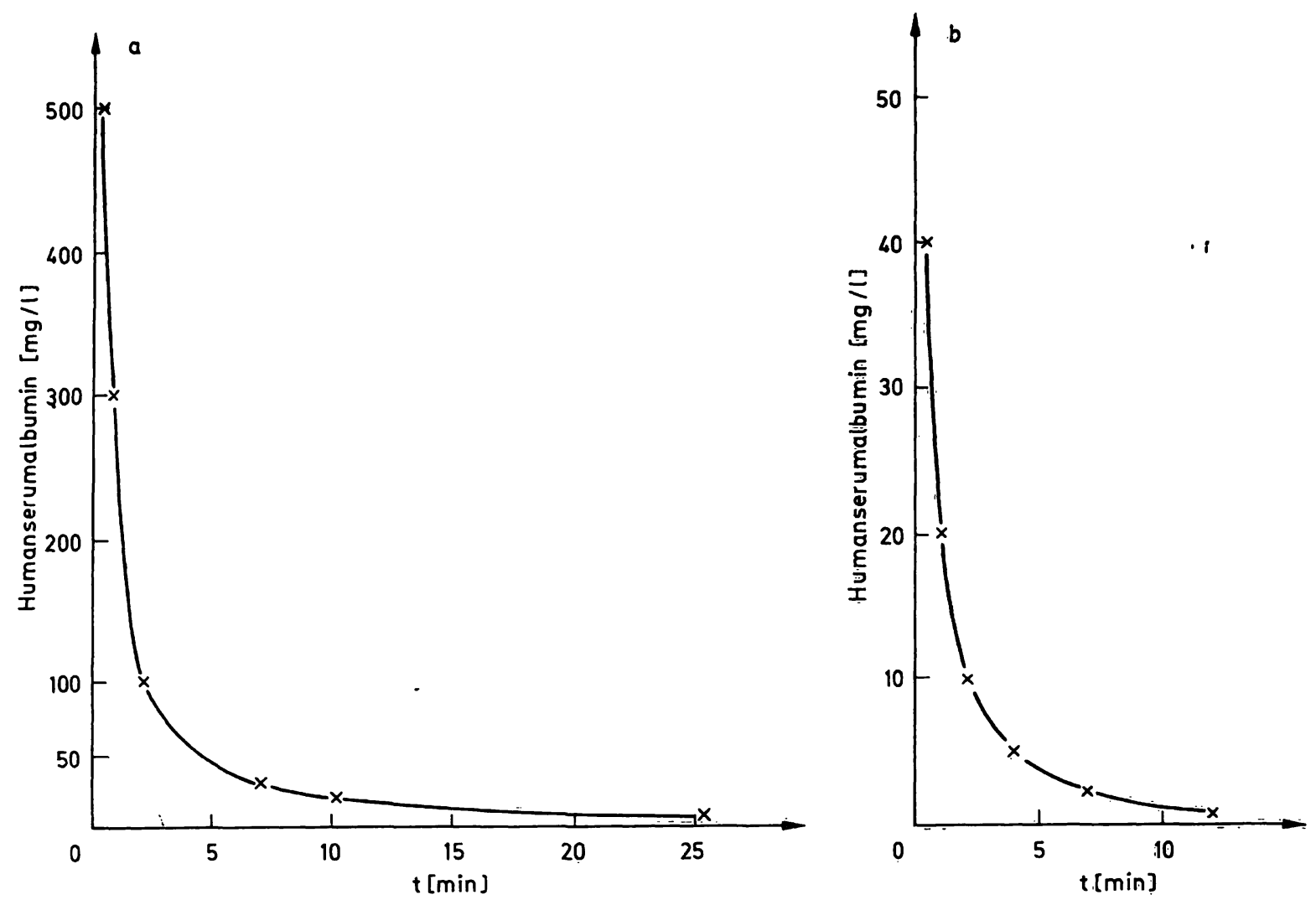

Abb. 6. Zeitbedarf für die Proteinbestimmung mit der Streulichtmethode, untersucht an Humanserumalbuminlösungen.

a) Methode $A(10-500 \mathrm{mg} / \mathrm{l})$,

b) Methode B (1-40 mg/l).

\section{Literatur}

1. Weichselbaum, T.E. (1946) Amer. J. Clin. Pathol. 16, 40-49.

2. Bradford, M. M. (1976) Anal. Biochem. 72, 248-254.

3. Henry, R.J., Cannon, D.C. \& Winkelman, J. W. (1974) Clinical Chemistry, Principles and Technics, S. 430-431, Harper and Row, New York.

4. Reiber, H. (1980) diese Z. 18, 123-127.

5. Thomas, L., Winkelmann, M., Michaelis, H. C. \& Walb, D. (1981) diese Z. 19, 203-208.
6. Hebell, Th. \& Schiwara, H. W., Publikation in Vorbereitung. 7. Markowetz, D. \& Munz, E. (1981) In: Methodische Fortschritte im Laboratorium. Kurzreferate (Rösler-Engelhardt, A. Hrsg.), S. 114, Verlag Kirchheim, Mainz.

8. Weber, M. N., Bitter, T. \& Scheler, F. (1983) Lab. Med. 7, $155-163$

9. Thomas, L. (1984) Labor und Diagnose, 2. Aufl., S. 334, Medizinische Verlagsgesellschaft Marburg/Lahn.

Dipl.-Chem. H. Kirchherr Dr. med. H.-W. Schiwara Straßburger Straße 19 D-2800 Bremen 1 